\title{
ON THE LOGIC OF NATURAL KINDS*
}

\author{
NINO COCCHIARELLA
}

Indiana University

\begin{abstract}
A minimal second order modal logic of natural kinds is formulated. Concepts are distinguished from properties and relations in the conceptual-logistic background of the logic through a distinction between free and bound predicate variables. Not all concepts (as indicated by free predicate variables) need have a property or relation corresponding to them (as values of bound predicate variables). Issues pertaining to identity and existence as impredicative concepts are examined and an analysis of mass terms as nominalized predicates for kinds of stuff is proposed. The minimal logic is extendible through a summum genus, an infima species or a partition principle for natural kinds.
\end{abstract}

A standard objection to quantified modal logic is that it breeds such reptiles of the mind as Aristotelian essentialism, "the doctrine that some of the attributes of a thing (quite independently of the language in which the thing is referred to, if at all) may be essential to the thing, and others accidental" ([5], p. 173f.). This objection has been criticized on one front by pointing out that none of the standard systems of quantified modal logic commit us to more than the meaningfulness of the non-trivial versions of the doctrine and that indeed we can, if we so choose, actually deny such versions in these systems (cf. [4]). A more heroic response, however, accepts these versions of the doctrine, at least when properly stated, and finds quantified modal logic the appropriate medium for its formulation. In what follows I shall attempt to formulate one such response, at least for the purpose of clarifying the general sort of logistic framework it presupposes if not also for exposing some of the more fascinating serpents that breed therein.

1. Natural Kinds. The properties that are essential to a thing are properties it necessarily has whenever it exists. Logically necessary properties, if there really be such, are of course essential to everything. These, however, are not the sort of property I have in mind in the version of the doctrine I want to discuss. Nor for that matter is logical necessity the sort of necessity I have in mind either. The clearest sort of framework for which I can make philosophical sense

*Received May, 1975.

Philosophy of Science 43 (1976) pp. 202-222.

Copyright (C) 1976 by Philosophy of Science Association. 
of logical necessity is logical atomism, and in that framework what is necessary for one object is necessary for all, an altogether trivial and uninteresting version of essentialism (cf. [2]).

The sort of essential properties I want to consider can best be called natural kinds. These properties, roughly speaking, are natural powers or capacities which things have to act, behave, function, etc., in certain specific determinate ways; and in this respect, they are causal structures grounding the law-like behavior of things of that kind.

I shall not explicitly assume in any of the logics that are formulated here that there are any natural kinds in reality, nor for that matter that there are any properties or relations in the world at all. Nevertheless, informally I understand natural kinds to include the various genera and species of plants and animals as well as the natural kinds of substances (in the chemical sense) such as gold, water, oxygen, etc. These latter might also be called "stuff," and, as indicated, I include as natural kinds of stuff compound substances such as water or salt as well as elementary substances such as gold, iron, oxygen, etc.

Whether heterogeneous stuff, on the other hand, such as wood, or alloys such as brass or bronze, or mixtures such as sugary water, dirty water, mud or dirt, etc., are also natural kinds of stuff I leave to the context of application to decide. For example, in the context of providing a linguistic analysis of mass terms such as is recommended in the extended theory described in Section 4 below where mass terms are nominalized predicates for natural kinds of stuff, it becomes convenient on purely linguistic grounds to construe predicates for alloys and mixed materials as predicates for natural kinds. However, for the more demanding purposes of natural science, an appropriate application of the system will probably require that predicate constants introduced for natural kinds must satisfy certain further theoretical constraints, particularly such as are indicative of their causal explanatory role within the framework of the science or sciences in question. In particular, however, I see no reason why some, if not all, of the monadic theoretical predicates of a given science might not designate natural kinds, though these may for the most part be kinds of "things," e.g., electrons or protons, rather than kinds of stuff.

By a "thing" in the scare-quoted sense of the word I mean an individual that is somehow a whole which is more than the sum of its constitutive parts or of the bits of stuff that make it up; that is, roughly what Aristotle would call a "primary substance." An animal or a plant, for example, is a "thing" that may change much of the material stuff that makes it up while still remaining the same animal 
or plant. A vein of gold in a mountain or the sodium chloride in my salt shaker, on the other hand, are individual bits of these natural kinds of stuff and in that regard they too are things (values of individual variables). But I do not consider them to be material "things" in the scare-quoted sense, i.e., as individual wholes which in their material mode of being are more than the mereological sum of the bits of stuff that make them up. An atom of gold or a $\mathrm{NaCl}$ molecule, however, are "things" qua being an atom of gold or a $\mathrm{NaCl}$ molecule. Qua being a bit of gold or a bit of salt an individual is not a "thing", in the scare-quoted sense (unless it is a minimal bit of that natural kind of stuff).

2. Physical or Nomological Necessity. As these brief remarks indicate, natural kinds are material and not logical essences, or to adopt Locke's terminology they are real and not nominal essences. Accordingly, the type of necessity or possibility which is appropriate in the logic of natural kinds is one pertaining to a material and not a logical modality. One view of such a modality is Aristotle's, for whom that is necessary which is always the case, and, dually, that which is possible is what is sometimes the case in the infinitude of time (cf. [3], chapter V). Such a view suffices for all that I have to say here regarding necessity and possibility.

A more liberal and controversial view, however, allows for a sense of necessity somehow determined by the laws of nature, whatever they may be. Possible worlds then are worlds in which the same laws of nature hold as hold here. Since worlds in which our laws of nature do not hold may be worlds in which what are natural kinds here need not be natural kinds there, such worlds are not worlds that are possible in our present sense of nomological possibility.

A more stringent intermediate view, on the other hand, takes a world to be a possible world relative to ours if and only if it is physically accessible to ours by some causal means (with light signals as an upper bound on causal influences). Possible worlds in other words are to be the alternative world-lines of the same cosmic system of world-lines to which our world-line(s) belong(s). And belonging to the same cosmic system in this sense entails our having the same laws of nature in the sense in which these are what hold invariantly through all the world-lines of our cosmic system.

Whatever the sense of natural or physical necessity we might choose in applications, the only assumption I shall make here is that the transworld relation of accessibility is both reflexive and transitive, i.e., that the strictly modal principles involved are those of the system $S 4$. This assumption is clearly justified under the causal transmission 
interpretation of necessity, i.e., where accessibility is through a chain of causal influences between world-lines; and under the more restricted temporal interpretation we would justify not only transitivity and reflexivity but symmetry as well (with respect to any given "local time"). The assumption amounts at best then to a restriction as to how liberal an interpretation nomological necessity will be allowed to have.

Aside from the strictly modal principles of $S 4$, an example of a mixed modal principle which will be valid in what I take to be the minimal system of a logic of natural kinds is the principle that an individual can belong to a natural kind only if being of that kind is essential to it, i.e., only if it must be an individual of that kind whenever it exists: ${ }^{1}$

$$
\text { (K1) } \forall x \forall^{k} F(\diamond F(x) \rightarrow \square[E !(x) \rightarrow F(x)])
$$

where $\forall^{k}$ is the universal quantifier ranging over natural kinds and ' $E$ !' is the predicate for material existence.

I do not assume that the predicate for material existence either stands for a material property of individuals or that it is a primitive sign of the logic of natural kinds. It does express or stand for a concept, however, whose analysis we will return to later. For now we only note that not every individual (qua value of a bound individual variable) is assumed to fall under that concept, i.e., it may be that some individuals do not now have a material existence.

Logical objects, of course, such as classes, if such there be, do not ever have a material existence, and hence these individuals trivially do not fall under that concept. But more than these, for example, where possibility is what is sometimes the case, either in our world or in one causally accessible to ours, past or future objects need not now have material existence, though they will still be reckoned here as individuals falling within the range of our quantifier. And if possibility is allowed the yet more liberal interpretation, we shall similarly allow for possible objects that do not ever materially exist in our cosmos, so long as they have material existence in some nomologically possible world.

Another mixed modal principle which I assume to be valid even in the minimal system is the assumption that the properties that are by:

${ }^{1}$ If the modal logic in question is $S 5$ rather than $S 4$, then $(K 1)$ can be replaced $\left(K 1^{\prime}\right) \forall x \forall^{k} F(F(x) \rightarrow \square[E !(x) \rightarrow F(x)])$

i.e., the principle that every natural kind to which an individual belongs is essential to that individual. 
natural kinds in our world are natural kinds in all physically possible worlds: ${ }^{2}$

(K2) $\forall^{k} F \square \exists^{k} G(F \equiv G)$

The quasi-identity sign ' $\equiv$ ' between predicate variables we take to be abbreviatory for necessary material equivalence:

$$
(F \equiv G)=_{d f} \square \forall x[F(x) \leftrightarrow G(x)]
$$

Unlike concepts, I understand material properties or relations to be no further discernible than through all their physically or nomologically realizable instances.

A further mixed modal principle which I take to be valid is the obvious assumption that every natural kind is physically or nomologically realizable:

(K3) $\forall^{k} F \diamond \exists x F(x)$

Aristotle made the stronger assumption that a natural kind cannot "be" unless it is actually realized, which together with (K2), has the consequence that every natural kind necessarily has an instance:

$$
\forall{ }^{k} F \square \exists x F(x)
$$

This latter thesis might perhaps even be acceptable if all it meant was that each natural kind must be realized in each physically possible world sometime or other. But that is not Aristotle's notion of necessity and his version seems to commit us to the view that somehow a natural kind cannot be unless it always has an instance, a consequence of which is the doctrine of the fixity of species.

Naturally, we intend not only to acknowledge the unfortunate extinction of many species but also the evolution of new species from old ones. It is even possible that a species may become extinct and then re-emerge or evolve again at a later time, as is indeed the case with the transuranic elements. Natural kinds as determinate causal structures or patterns in nature have a material potentiality to be realized, even when there are no individuals of that kind, no less than do material objects have their own form of potential existence prior to their actual coming-to-be. Thus when nature finished with the earth as we know it today it contained 92 chemical elements or elementary substances with uranium as the heaviest naturally occurring on earth. Whether atoms of transuranic elements exist in

${ }^{2}$ This principle has the commutative law

$$
\square \forall^{k} F \phi \rightarrow \forall^{k} F \square \phi
$$

as one of its consequences. 
the universe outside the earth is for the most part unknown. But even if they do not, these elements are no less natural kinds of elementary substances than are those whose atoms abound in nature. Indeed, so much is known about these elementary causal structures that transuranic atoms have actually been generated in accelerators by bombardment of other elements with heavy nuclei. The latest such elementary substance to have atoms generated in this manner is element 106, whose half-life is 0.9 seconds.

Before concluding this section I should perhaps note that the modal operator $\diamond$ for physical or nomological possibility does not represent anything like Aristotle's notion of physical capacity or potentiality. Thus, assuming that oak trees are a natural kind and that an oak tree acorn is not an actual but only a potential oak tree, then, by $(K 1)$, there is no physically or nomologically possible world, i.e., a world where the laws of nature are as they are here, in which $i t$, the acorn, is an oak tree. Similarly, assuming that humans are a natural kind and that a human fetus is not an actual but only a potential human, then there is no physically or nomologically possible world where it, the fetus, is a human.

Where possibility is what is sometimes the case, the principle (K1) may lead to some confusion about the nature of identity. Thus corresponding to every human and to every oak tree there did exist a human fetus and an oak tree acorn which became that human, and that oak tree, respectively. But 'becoming', as Aristotle says, has many meanings (Physics I, 7, 190a 32), and the coming-to-be of an oak tree from an acorn or of a human from a fetus need not-and cannot, if oak trees and humans are natural kinds-be interpreted as a coming-to-be of the same individual or material unity changing in one or more of its non-essential properties. An alternative interpretation is that it is one kind of individual being which is generated from another causally related kind, where the transmutation of the one material unity into the other is a consequence of the laws of nature. Similarly, if atoms are atoms only qua being atoms of a natural kind of an elementary substance, then when Rutherford transmuted a sample of nitrogen into oxygen by bombarding the former with alpha particles, atoms of nitrogen became atoms of oxygen, even though it is not the case that one and the same atom is at one time a nitrogen atom and at a later time an oxygen atom. Nor under similar assumptions is a neutron a composite of a proton and an electron, even though it has the physical capacity or potentiality for disintegrating into such; nor of course is a neutron, which upon disintegration becomes an electron and a proton, identical with either that electron or that proton. In these matters it is science and not logic that is at issue. 
3. The Conceptual-Logistic Background. The principles $(K 1)-(K 3)$ cited above along with the two auxiliary schemas

(K4) $\forall^{k} F(\phi \rightarrow \psi) \rightarrow\left(\forall^{k} F \phi \rightarrow \forall^{k} F \psi\right)$

(K5) $\forall F \phi \rightarrow \forall^{k} F \phi$

and the $S 4$ modal principles characterize what I take to be the minimal system of a logic of natural kinds, at least when these are embedded in a logical framework encompassing the valid schemas of first-order logic with identity and some minimal assumptions regarding predicate quantifiers. Subsequently, in sections 7-8, we shall consider certain extensions of this minimal system, i.e., extensions determined by additional mixed modal principles regarding natural kinds. At the present juncture, however, I shall attempt to explain how I understand the conceptual-logistic background implicit in all of these systems. A purely set-theoretic semantics or model theory characteristic of these systems, i.e., for which completeness theorems are forthcoming, can be found in the appendix.

In the way of logical grammar, I shall assume the usual terminology and distinctions of pure second-order modal logic with identity, except for the addition of the universal and existential quantifiers for natural kinds. ${ }^{3}$ Applications of the pure grammar will in general be restricted to the introduction only of predicate constants and the postulates intended for them, among which may be included meaning postulates. It is assumed that every wff (well-formed formula) which is an instance of a valid schema of first-order logic with identity is also valid here. It is not assumed, however, that all the wffs that are instances of valid schemas of standard second-order logic are also valid here.

In general, I shall speak of a predicate or open $w f f$ as expressing or standing for a possible (first-level) concept relative to certain of the free individual variables occurring therein (as argument indicators). By the possibility of a concept I mean the capacity humans (or thinkers in general) have to formulate and exercise that concept in either overt or covert acts of judgment, regardless of whether the concept has been formulated and exercised thus far or not. I assume in this regard that every possible (first-level) concept is in principle expressible by some predicate or open wff, whether of the pure or applied form of the logical grammar. However, other than the minimal metalinguistic condition that different expressions for the same concept must be analytically equivalent, I shall leave the choice and decision for all

${ }^{3}$ We use ' $x$ ', ' $y$ ', ' $z$ ', $\ldots$, for individual variables and ' $F^{n}$ ', ' $G^{n}$ ', ' $H^{n},, \ldots$, for $n$-place predicate variables. We delete the superscript when the context makes clear the degree of the predicate variable in question. 
further conditions regarding the "individuation" of concepts to the particular contexts of application.

This "pragmatic" position regarding the individuation of concepts is possible in the present framework, it should be noted, precisely because it is not concepts that our predicate quantifiers range over but physical or material properties and relations instead. However, notwithstanding the fact that they are not the values of the bound $n$-place predicate variables, $n$-ary concepts may nevertheless be viewed informally as the values of free $n$-place predicate variables, since it is not assumed in the present framework that whatever is the value of a free $n$-place predicate variable is also the value of a bound $n$-place predicate variable. In this respect, concepts differ from material properties and relations somewhat in the way that merely possible objects differ from existing objects in those modal logics which quantify only over the individuals existing in the world in question while allowing free individual variables to have objects existing in other worlds as their values. In the present framework, we take the latter distinction, but not the former, to be without merit. That is, in the present framework the distinction between the conceptual and the real applies only to predicable entities, i.e., to concepts and material properties and relations, and not at all to individuals. ${ }^{4}$

Now it will no doubt be noted that this distinction between concepts on the one hand and properties and relations on the other is without much import in the present logical grammar if we assume the general comprehension principle:

$$
\text { (CP) } \exists F \square \forall x_{1} \ldots \forall x_{n}\left[F\left(x_{1}, \ldots, x_{n}\right) \leftrightarrow \phi\right]
$$

for all open $w f f s \phi$, where ' $x_{1}$ ', ., ' $x_{n}$ ' are among the distinct individual variables occurring free in $\phi$ and ' $F$ ' is an $n$-place predicate variable not occurring (free) in $\phi$. For in that case we would be assuming the existence of a property or relation corresponding to each $n$-ary concept indicated by a free $n$-place predicate variable; and, accordingly, without incorporating further logistic devices for distinguishing between the conceptual and the real, we would in effect be identifying concepts with their corresponding properties and relations. ${ }^{5}$

However, in so far as predicate quantifiers are understood to range

${ }^{4}$ This is not to say of course that the notion of a possible object which never exists at any (local) time of any world-line of our cosmos, i.e., a merely nomologically possible object in our liberal sense, is also not without merit.

${ }^{5}$ In a somewhat richer system where, e.g., a logical device for something like Sellars' dot-quotes has been incorporated and systematically developed we might allow $(C P)$ for special predicate quantifiers ranging over concepts as predicate senses or linguistic types in a sense akin to Sellars' (cf. Sellars [6]). These predicate quantifiers of course 
over physical or material properties and relations, the assumption that $(C P)$ should be valid is completely unfounded and seems in general to be based upon a confusion between the conceptual and the real. On the other hand, the fact that $(C P)$ is not valid in general should not be thought to imply that material properties and relations are in some sense "ontologically simple," particularly since special instances of $(C P)$ involving rather complex and perhaps even impredicative comprehending wffs will usually be assumed in particular contexts of application. ${ }^{6}$

Thus, whether there really are any material properties or relations, and, in particular, whether there are any natural kinds in the world or not is a matter for science and not for logic to decide. Accordingly, while it is assumed (even if only as vacuously true) that every material property or relation of our world is a material property or relation in every physically or nomologically possible world:

$$
\forall F^{n} \square \exists G^{n}(F \equiv G)
$$

it is not also assumed, at least not on logical grounds alone, that there is a material property or relation corresponding to any possible concept, i.e., the consistency of the following schema is allowed in the present framework:

$$
\sim \exists G^{n}\left(F^{n} \equiv G^{n}\right)
$$

We might note in regard to the consistency of this last wff that even though $n$-place predicate quantifiers do not range over possible $n$-ary concepts, we can simulate a weak form of such quantification indirectly by a metalinguistic quantification over $n$-place predicates or open wffs (relative to $n$ individual variables specified as argument indicators), particularly since it is assumed that every possible concept is in principle expressible by a predicate or open wff. Thus while

must then be distinguished from those ranging over material properties and relations as potentially real material forms in nature.

The fact, incidentally, that $(C P)$ in such a richer system allows for the specification of impredicative concepts, i.e., concepts whose specification involves predicate quantifiers, would not be seen as objectionable in the framework of a holistic ralitionalist theory of mind. And the fact that the mind has the innate capacity to formulate and exercise such concepts may well be the necessary basis for the principles needed to account for initial language acquisition.

${ }^{6}$ The notion of impredicativity applies primarily to $w f f s$ and secondarily to concepts as the conceptual counterparts of open wffs. I do not assume, on the other hand, that it has any application to material properties or relations even when these are theoretically projected or posited only through impredicative concepts. This is because material properties and relations do not depend on the structure of thought the way concepts do. 
$(C P)$ is not taken to be a valid principle, the (metalinguistic) substitution rule:

(S) if $\vdash \psi$, then $\vdash S_{\phi}^{F\left(x_{1}, \ldots, x_{n}\right)} \psi \mid$

is presumed to be valid in all our systems. This rule, as noted, while not equivalent to $(C P)$, nevertheless allows for the requisite weak sense of quantification over concepts.

In concluding this section, it should perhaps be noted once again that while concepts are assumed to depend on the cognitive capacity of the human mind, material properties and relations, and natural kinds in particular, are presumed to be potentially real material forms, physical states, or causal structures in nature. Accordingly, if to the concept horse there corresponds a natural kind, a causal ground or basic genetic form which is found in all and only horses, then the latter, unlike the concept to which it corresponds, depends no more on the cognitive capacity of the human mind then do the horses it is found in. Moreover, no presumption is made here that the conceptual content of the concept horse, at least as we exercise that concept in ordinary language today, somehow contains or entails the conceptual counterparts of any or all of the material properties that are causal consequences of belonging to that natural kind, as though to have the concept as a realized cognitive capacity were to suffice to know the details of the basic genetic plan for being a horse.

4. Natural Kinds as Classes and Mereological Sums. Despite the fact that concepts are cognitive capacities and properties and relations are potentially real material forms, both, from the logical point of view of the present framework, are presumed to be entities of a strictly predicable nature, i.e., both are semantically associated only with predicates or open wffs. It is intended, however, that their strictly predicable nature extends beyond the parochial confines of our present rules of well-formedness. For it is our view that neither concepts on the one hand nor properties and relations on the other have an individual nature, i.e., neither can fall under concepts or have properties and stand in relations in anything at all like the sense in which individuals do (though they may fall within second-level concepts or material structures in a related analogical sense).

I do not deny that the opposite appears to be the case in the surface grammar of ordinary language. Nor do I intend to cease exploiting that appearance for the practical and informal expository purposes of this paper. Nor, moreover, do I wish to deny that there may well be a genuine philosophical insight behind that surface appearance; indeed one very much to the point regarding the real 
conceptual distinction between our two types of natural kinds, though it certainly is not what either platonists or nominalists have traditionally taken it to be.

In the way of an explanation of these remarks let us consider the possibility of extending our present rules of well-formedness so as to allow for the occurrence of nominalized predicates, i.e., occurrences of predicates (or of open $w f f s$ if the $\lambda$-operator is also available) occupying positions now occupied only by individual variables. We cannot of course interpret such occurrences as referring to the concepts or properties which the same predicates express or stand for when occurring in predicate positions, at least not if concepts and properties really do not have an individual nature either in the subjective structure of thought or in the objective structure of the world. Rather, following Frege's original insight on this matter, we are to interpret nominalized predicates as referring to certain individuals that are systematically correlated in the structure of thought with the concepts expressed by predicate position occurrences of these same predicates. Adopting Frege's terminology, I shall call such individuals concept-correlates.

Now within the sort of logical framework which I have been describing, the concept-correlates of concepts of natural kinds of stuff as opposed to those of natural kinds of "things" are of particular interest. For from the point of view of this framework, as extended so as to allow nominalized predicates, the real logical or conceptual difference between these different types of natural kinds is a difference not in their predicable nature, a thesis which even the more restricted grammar maintains but is unable to explain, but in the individual nature of the type of individual with which each is correlated. Thus, on this view, the individual which is correlated with the concept of a natural kind of "thing" is the class of all the individuals of that kind, an individual whose individuality is abstract or logical and therefore not of that or any other natural kind. The individual which is correlated with the concept of a natural kind of stuff, on the other hand, is on this view the spatio-temporally scattered individual which is the mereological sum of all the individuals of that kind, an individual which is itself of that natural kind and whose individuality is therefore of a material nature.

As correlates of concepts of natural kinds, these different types of individuals bring with them in the structure of thought certain dyadic or relational concepts under which they stand to all the individuals falling under the concept of the kind of which they are correlates. Thus all the individuals of a natural kind of "thing" belong to or are members of the class of things of that kind; and all the individuals of a natural kind of stuff are bits of the mereological 
sum of these individuals. It is this relational terminology and the individual nature of these concept-correlates which have become conflated in the surface grammar of ordinary language with the terminology of predication and the strictly predicable nature of concepts or properties.

However this conflation has come about, and independently of whether we accept the metaphysics of this extended view, I shall myself continue to exploit the convenience of this mixed terminology by speaking of the individuals of a natural kind as belonging to or being bits of the kind itself. In this way I shall speak as though there were two forms of predication for each type of natural kind-indeed, as though predication were itself a relation between individuals.

From the conceptual point of view of this grammatical extension of the minimal system, the fundamental error of both platonism and nominalism is that each conflates properties or concepts with their correlates and thereby confuses predication either with membershipor exemplification, a supposed non-extensional counterpart of membership-on the one hand, or with the mereological bit-of relation on the other. From the perspective of even the minimal system, however, predication is not a relation, or at least not a relation between individuals, though it may be an (unequal-leveled) second-level dyadic concept between individuals and (first-level) concepts, some of the latter of which may have corresponding (material) properties. ${ }^{7}$

5. Identity. I should like to maintain the general thesis that logical or formal concepts under which individuals fall, i.e., formal first-level concepts, are all of them derivative concepts based upon purely formal second- or higher-level concepts. On this view, as I understand it, formal first-level concepts, unlike most of their descriptive counterparts, ${ }^{8}$ enter into our conceptual framework in an essentially

${ }^{7}$ The terminology of levels throughout is essentially that of Frege. While many of the views expressed here are reminiscent of Frege's, I do not assume that concepts or properties and relations have a strictly predicable nature in anything at all like the sense he intended, viz., that they are unsaturated functions from individuals to truth-values. The model theory in the appendix, however, does use set-theoretic functions of this sort as mathematical counterparts of concepts and the latter as counterparts of their corresponding properties and relations when such are presumed to exist in the model in question.

${ }^{8}$ The descriptive concepts that are strictly theoretical constructs of a scientific theory are in my view only impredicatively specifiable in terms of the Hilbert $\epsilon$-operator (for predicate variables) applied to the theoretical postulates of the theory (cf. Carnap [1]). Since the Ramsey-sentence of these postulates with their theoretical predicates so specified is then a consequence of the theory, these are, accordingly, descriptive concepts for which we posit corresponding material properties and relations in the world. Not all, or perhaps not even any, of the remaining so-called observational 
impredicative manner. Such a mode of entry explains in particular why there are and can be no material properties or relations corresponding to these concepts. ${ }^{9}$

Unfortunately, however, our non-allowance of $(C P)$ leaves indiscernibility inadequate as a basis for the substitutivity of identity, and for this reason we have taken the latter as a logical primitive, as though the dyadic concept it stands for were a simple relational concept in the structure of thought. Of course, precisely because of the substitutivity of identity even in contexts with predicate quantifiers it is clear that identity entails and therefore contains impredicative content.

I have already noted that the concept of identity may lead to some confusion in the context of a logic of natural kinds of "things," as when an acorn becomes an oak tree, or when a fetus becomes a human. This possibility of confusion is no less in the context of natural kinds of stuff, as when a gold nugget becomes a ring, is melted down and becomes a nugget once again, or when a sample of salt becomes samples of sodium and chlorine gas after an electric current is passed through it. And artifacts have their identity "crises" too, as with the wooden ship all the boards of which are removed and replaced one by one over a period of time and later put together again to form another (?) or the same (?) original ship.

What these and similar conceptual puzzles show us is that identity is a complex formal concept inextricably bound to our various principles of individuation. Such principles, however, ultimately depend in their different formulations on different conceptual or metaphysical schemes. Independently of such a scheme and principles of individuation integral to that scheme there just is no pure and simple concept of identity, despite our formalistic pretensions to the contrary.

From a strictly conceptual point of view, for example, the principles of individuation for logical objects, e.g., classes, are perhaps the clearest we can formulate, at least in so far as these objects have a purely formal or conceptual mode of being. Thus the principle of individuation for classes is compactly stated in the extensionality law: classes are identical when and only when they have the same members. Membership in a class, however, with or without the explicit

concepts of the theory need also have a material property or relation corresponding to them in the world. This ontological asymmetry between theoretical and observational concepts, like their supposed converse epistemological asymmetry, is reminiscent of the traditional distinction between concepts of primary and secondary qualities.

${ }^{9}$ The same explanation does not apply to the theoretical constructs that are only impredicatively specified in terms of the Hilbert $\boldsymbol{\epsilon}$-operator (for predicate variables) precisely because the predicate that expresses or stands for the construct is bound by the operator. 
presence of the variable-binding (second-level) class-abstraction operator, is an impredicative dyadic concept through which classes have their peculiarly dependent or purely relational mode of being. Viewed in this respect the impredicative character of class-identity stands out as rather obvious.

Our principles of individuation for bits of stuff, on the other hand, are a mixed bag of conceptual rules consisting in part of "individuating standards," as when we speak of a body or cupful of water, or a vein or nugget of gold, as opposed to water or gold simpliciter; and in part also of certain mereological laws as that bits of a natural kind of stuff are identical (qua being bits of that natural kind) when and only when every (minimal) bit of the one is a (minimal) bit of the other. Here the suppression not only of individuating standards but of the kinds of stuff implicit in mereological laws allows us again to overlook the complex and impredicative character of identity.

The individuation of a material "thing" in our scare-quoted sense is a more complex matter, however, for here we confront artifacts as well as "things" belonging to a natural kind. Principles of individuation for artifacts refer us not to the material nature of such a "thing" so much as to certain human standards, e.g., implicit or explicit normative rules, concepts or the like, regarding the social function, purpose, or moral or aesthetic significance of that "thing." In contrast, the materially real individuation of a natural kind of "thing" depends not at all on our normative standards but on a materially real form or structure constituting the individual material nature of that "thing."

One possible extension of our present logical grammar wherein we might attempt a formal representation of such individual natures is the addition of quantifiers binding second-level variable-binding formula operators such as the universal or existential quantifiers for individual variables. The free occurrence of such an operator expresses or stands for what we, following Frege, have been calling second-level concepts (within, not under, which first-level concepts fall). Of course like first-level concepts, not all second-level concepts would have a material form or structure corresponding to them in the world. Thus, while associated with every individual there is, on the one hand, a second-level concept within which fall all and only those first-level concepts under which that individual falls - a second-level concept which, adopting Duns Scotus' terminology, we might call the haecceity of that individual-there would exist in the world, on the other hand, material forms or structures corresponding only to those second-level concepts that are the haecceity of a "thing", belonging to a natural kind. Such a material form or structure would causally account for the natural kinds to which that individual belonged 
as well as the special physical mode of being in which that individual is a whole which is more than the sum of its parts or of the bits of stuff that make it up-a whole, that is, which somehow remains the same individual even while changing some of its parts or the bits of stuff that make it up. The haecceity of an artifact or of a (non-minimal) bit of stuff, e.g., a bit of gold, or of a logical object, e.g., a class, on the other hand, would have no such material structure corresponding to it.

Such are the ways in which a fuller and more explicit framework than the minimal system we describe here might approach the concept of identity. The minimal system, nevertheless, is not without its conceptual utility however schematic that might be, and for that reason we leave identity as it is.

6. Material Existence. The impredicative character of the concept of physical or material existence is altogether another matter, however. For in this case I believe we have available just the sort of distinction in terms of which that concept might be analyzed and more clearly represented.

That there is no one physical state or material property corresponding to this concept of existence is of course an old and familiar thesis and one with which I have no quarrel here. That is, it is assumed in the present framework that among the material properties that form and determine the existence of a physical or material object, it is not the case that there is one which corresponds to our concept of material existence.

Nevertheless, neither this concept nor its complement is without its conceptual utility. For it is also not assumed here that this concept coincides, either necessarily or contingently, with the concept of the being of an individual as the value of a bound individual variable.

Non-existence in the material mode, in other words, is not a necessarily vacuous concept. And if classes or other logical objects are presumed to be among the individuals, then it never is a vacuous concept. For a logical object belongs to no natural kind and falls under no concept to which there corresponds a material property or relation.

In addition, past or future but presently non-existing objects, i.e., objects which do exist in the material mode either in the past or the future, or, on the liberal (and controversial) view, objects which could (in the sense of nomological possibility) so exist but which in fact never do: one and all fall under the concept of non-existence while still being the same individuals they are when they do exist, though of course not falling now under all the same concepts they 
fall under then. And in this sense there is a subject of change which remains identical with itself even in the case of substantial coming-tobe (generation) or ceasing-to-be (annihilation) of a physical or material object, though of course, given conservation of matter and energy laws, this is not a coming-to-be out of, or a ceasing-to-be into, nothing.

Relative now to concepts corresponding to which there are material properties or relations, presently non-existing objects, not now falling under any of these concepts, are one and all indiscernible each from the other. That is, in terms of the material properties which determine the world's present material existence in the sense of the totality of physical or material objects presently existing in the world, these non-existing objects are one and all indiscernible. Yet each nonetheless is discernible from everything else at least in terms of the temporal, causal and relational concepts to presently existing objects under which it now falls. In other words, all that their failure to exist now in the material mode amounts to is their failure now to have any material property. What this suggest, accordingly, is an analysis of material existence as the possession of some physical or material property:

$$
E !(x)={ }_{d f} \exists F F(x)
$$

Standing in a material relation to presently existing objects, incidentally, need not itself constitute a material property or a monadic physical state. To insist otherwise is to confuse logical relationships between concepts with potentially real material forms or causal structures. Accordingly, if it is possible that an existing physical object might stand in various material relations while not also having itself any material property or being in any monadic physical state, then the above definition would seem to be deficient. I assume that just this sort of situation is not possible, however, since otherwise a material object, and even the world as a whole, might then have a purely relational mode of being.

Another assumption related to this is that the mode of being of an object that presently does not but could materially exist, and in particular that does exist in the past or future, need not be merely an intentional mode having no objective content independent of our conceptual ability to refer to it and other such non-existing objects. In particular, I do not see why some of the temporal or causal relational concepts under which non-existing objects now fall or stand to presently existing objects might not have material relations corresponding to them so that while such presently non-existing objects have now no material or physical properties, they nevertheless might stand now in certain material relations to presently existing objects. A neutron which became an electron and a proton is strictly speaking no longer 
in existence. Yet its disintegration into the presently existing electron and proton may very well constitute a physical or material relation which obtains even now between it and its resulting electron-proton pair. Other similar material relations might obtain even now between a past but no longer existing acorn and a presently existing oak tree or between what was a human fetus and what is today a human being. And naturally, if such material relations are not to be excluded, then we cannot maintain that standing in a material relation alone suffices to ground the present existence of a material or physical object.

If the above definition is acceptable, then, by (K5), whatever belongs to a natural kind exists at the time or world of such belonging, and therefore by $(K 1),(K 2)$ and elementary transformations, an individual can belong to two natural kinds only if it belongs to the one when and only when it belongs to the other:

$$
\text { (K6) } \forall x \forall^{k} F \forall^{k} G(\diamond F(x) \wedge \diamond G(x) \rightarrow \square[F(x) \leftrightarrow G(x)])
$$

Since it is intended that $(K 6)$ is to be valid even in the minimal system, it is to be taken as an additional axiom if the above definition for material existence, or rather the ontological claim on which it depends, is not found acceptable.

7. The Summum Genus and Infima Species Principles. In turning now to some of the possible extensions of the minimal system which do not involve any changes in the logical grammar, let us say that one monadic concept is subsumed under or subordinate to another if it is necessarily included in the other. The subsumption or subordination is said to be proper if the latter concept is not also subsumed under the former:

$$
\begin{aligned}
& (F \leq G)={ }_{d f} \square \forall x[F(x) \rightarrow G(x)] \\
& (F<G)={ }_{d f}(F \leq G) \wedge \sim(G \leq F)
\end{aligned}
$$

The first and perhaps prima facie most plausible principle beyond those of the minimal system is the principle that if an individual belongs to any natural kind at all, then there is one to which it belongs which has subordinate to it every natural kind to which it belongs:

$$
\text { (K7) } \forall x\left[\exists^{k} F F(x) \rightarrow \exists^{k} F\left(F(x) \wedge \forall^{k} G[G(x) \rightarrow G \leq F]\right)\right]
$$

As causal material structures that are not limited by our capacity to formulate concepts to which they may correspond, on the other hand, I do not see why natural kinds must satisfy this summum genus principle, even allowing that the family of natural kinds to which an individual belongs forms a chain of subordination to one another. 
That is, I do not see why nature must conform to our finitary classificatory schemes by having an upper bound on every such possible chain of subordination between natural kinds.

Similarly, I do not see why nature must impose a lower bound or an infima species on the natural kinds to which an individual may belong. Yet this principle too has been traditionally maintained:

(K8) $\forall x\left[\exists^{k} F F(x) \rightarrow \exists^{k} F\left(F(x) \rightarrow \forall^{k} G[G(x \rightarrow F \leq G])\right]\right.$

Of course, if it is possible for an individual to belong to but one natural kind, then it is possible for the same natural kind to be both an infima species and a summum genus.

8. The Partition Principle for Natural Kinds. The idea that the family of natural kinds to which an individual belongs determines a chain of subordination of these kinds one to another is itself a consequence of the principle that if two natural kinds are not nomologically disjoint, then one must be subsumed under the other:

$$
\text { (K9) } \forall^{k} F \forall^{k} G(\diamond \exists x[F(x) \wedge G(x)] \rightarrow F \leq G \vee G \leq F)
$$

This principle does seem to be a rather natural assumption and appears to lead to a more plausible extension of the minimal system than do either $(K 7)$ or $(K 8)$. In addition, it yields as a consequence a partition principle for natural kinds according to which natural kinds that are subsumed under the same immediate genus are either nomologically identical or nomologically disjoint:

$$
\begin{aligned}
& \forall^{k} F \forall^{k} G \forall^{k} H\left[F<H \wedge G<H \wedge \forall^{k} H^{\prime}\right. \\
& \left(F<H^{\prime} \rightarrow H \leq H^{\prime}\right) \wedge \forall^{k} H^{\prime}\left(G<H^{\prime} \rightarrow H \leq H^{\prime}\right) \\
& \rightarrow \sim \diamond \exists x(F(x) \wedge G(x)) \vee(F \equiv G)]
\end{aligned}
$$

It is not assumed in $(K 10)$, on the other hand, that every natural kind which is a species, i.e., which is properly subsumed under another natural kind, has an immediate genus under which it is subsumed. In so far as natural kinds are potentially and nomologically real determinate forms or material structures not dependent on our conceptual classifications, I do not see why the subsumption relation between natural kinds might not be dense, or even continuous for that matter.

Finally, in connection with the partition principle, another candidate for a logic of natural kinds is the principle that every genus is the sum of its species:

(K11) $\forall^{k} F\left[\exists^{k} G(G<F) \rightarrow \square \forall x(F(x)\right.$ $\left.\left.\leftrightarrow \exists^{k} G[G(x) \wedge G<F]\right)\right]$

Naturally, this principle can be easily formulated so as to apply only 
to genera that have immediate species and which are then posited to be the sum of these immediate species, i.e., where ' $G<F$ ' in (K11) is replaced by ' $G<F \wedge \forall^{k} H(G<H \rightarrow F \leq H)$ '.

9. Concluding Remarks. The above are only some of the more obvious principles that come to mind in the development and application of a logic of natural kinds. My objection to including them within the minimal system is based solely upon the rather strong sense of independence from the structure of thought (and therefore of "logic") which I assume natural kinds to have. Of course, in certain restricted contexts or for specialized applications these principles, and perhaps others as well, may be fully justified and used accordingly.

There are of course other developments and applications which I have not touched upon at all in this paper, e.g., the analysis of causal counterfactuals as based upon natural kinds or of a comparative similarity relation between individuals in terms of the natural kinds they share, etc. Our interests in these sorts of developments or applications should, it is hoped, vindicate at least to some extent the ontology of natural kinds as causal or nomological essences. In any case, such reptiles of the mind as these are taken to be by some philosophers seem hardly poisonous or deadly at all.

Finally, there is the sort of application suggested in section 4 for extending the logic of natural kinds to include nominalized predicates so as to provide a general analysis of the logic and ontology of mass terms. I have only hinted throughout this essay at how this richer framework might be developed, and though I do have some further suggestions which I have not gone into here, it is hoped that perhaps others will also take up the clarion call to defend this rather fascinating serpent of the mind.

\section{APPENDIX}

1. Syntax:

As logical particles I shall use only $\sim, \rightarrow, \square,=, \forall$ and $\forall^{k}$ for the negation, (material) conditional, necessity, identity and universal quantifier signs. The quantifier $\forall$ is affixed to both individual and predicate variables while $\forall^{k}$ is affixed only to monadic predicate variables. Atomic wffs are expressions either of the form of an identity, $\alpha=\beta$, where $\alpha, \beta$ are individual variables, or of the form $\pi\left(\alpha_{1}, \ldots, \alpha_{n}\right)$, where $n \in \omega-$ $\{0\}, \pi$ is an $n$-place predicate variable and $\alpha_{1}, \ldots, \alpha_{n}$ are individual variables. The class of wffs is the smallest class containing the atomic wffs and such that $\sim \phi, \square \phi$, $(\phi \rightarrow \psi), \forall \alpha \phi, \forall \pi \phi, \forall^{k} \sigma \phi$ are all wffs whenever $\phi, \psi$ are $w f f s, \alpha$ is an individual variable and $\pi$ is an $n$-place predicate variable, for some $n \in \omega-\{0\}$, and $\sigma$ is a 1-place predicate variable.

2. Semantics:

A natural kind model (NK-model) is a structure of the form $\left\langle D, I, R,\left\langle F_{n}\right\rangle n \in \omega\right.$ $\left.-\{0\},\left\langle K_{u}\right\rangle_{u \in D}\right\rangle$, where (1) the universe $D$ of possibilia, and the set $I$ of possible 
worlds are non-empty; (2) $R$ is a reflexive and transitive relation on $I$; (3) for all $n \in \omega-\{0\}, F_{n} \subseteq\left(\mathscr{P}\left(D^{n}\right)\right)^{I}$; (4) for all $u \in D$ : (i) $K_{u} \subseteq F_{1}$, i.e., every natural kind to which the individual $u$ belongs is a material property in the model, (ii) for all $X \in K_{u}$, for some $i \in I, u \in X_{i}$, i.e., a property is a natural kind to which $u$ belongs only if $u$ can have that property, (iii) for all $X$, for all $v \in D$, for all $i \in I$, if $X \in k_{v}$ and $u \in X_{i}$ then $X \in K_{u}$, i.e., every natural kind to which $u$ can belong is a natural kind of $u$, and (iv) for all $X \in K_{u}$, for all $Y \in F_{1}$, for all $i \in I$, if $u \in Y_{i}$, then $u \in X_{i}$, i.e., $u$ belongs to each of its natural kinds whenever it has a material property (i.e., whenever it exists).

The indexed set $\left\langle F_{n}\right\rangle_{n \in \omega-\{0\}}$ specifies the (set-theoretical counterparts of the) material properties and relations in the $N K$-model. The indexed set $\left\langle K_{u}\right\rangle_{u \in D}$ specifies, for each $u \in D$, the set of natural kinds to which $u$ belongs (when it exists). If $u$ never belongs to any natural kind, e.g., if $u$ is an artifact or a logical object, then $K_{u}$ is empty. But if $K_{u}$ is not empty, then there is at least one possible world where $u$ materially exists and at which it belongs to all of its natural kinds. Finally, the set of individuals which exist in the material mode at a possible world $i \in I$ is the set $\left\{u \in D\right.$ : for some $\left.Y \in F_{1}, u \in Y_{i}\right\}$.

The additional clauses needed to validate $(K 7),(K 8),(K 9)$, from which $(K 10)$ follows, and $(K 11)$, respectively are:

$(4 / v)$ if $K_{u}$ is not empty, then for some $X \in K_{\mathrm{u}}$, for all $Y \in K_{u}$, for all $i \in I$, $Y_{i} \subseteq X_{i}$;

(4/vi) if $K_{u}$ is not empty, then for some $X \in K_{u}$, for all $Y \in K_{u}$, for all $i \in I$, $X_{i} \subseteq Y_{i}$

(4/vii) for all $X, Y \in K_{u}$, either for all $i \in I, X_{i} \subseteq Y_{i}$ or for all $i \in I$, $Y_{i} \subseteq X_{i}$

(4/viii) for all $X \in K_{u}$, if for some $Y \in K_{u}$, for all $i \in I, Y_{i} \subseteq X_{i}$ and $Y \neq X$, then for all $i \in I, X_{i}=\left\{v \in D\right.$ : for some $Y \in K_{v}, v \in Y_{i}$ and $Y \neq X$ and for all $\left.j \in I, Y_{j} \subseteq X_{j}\right\}$

An assignment in an NK-model $\mathbf{A}$ (as described above) is a function a with the set of individual and predicate variables as domain and such that $\mathbf{a}(\alpha) \in D$, whenever $\alpha$ is an individual variable, and $\mathbf{a}(\pi) \in \mathscr{P}\left(D^{n}\right)^{I}$, whenever $\pi$ is an $n$-place predicate variable. Satisfaction in $\mathbf{A}$ by a at a possible world $i \in I$ is defined recursively as follows: a sat $\alpha=\beta$ in $\mathbf{A}$ at $i$ iff $\mathbf{a}(\alpha)=\mathbf{a}(\beta)$; $\mathbf{a}$ sat $\pi\left(\alpha_{1}, \ldots, \alpha_{n}\right)$ in $\mathbf{A}$ at $i$ iff $\left\langle\mathbf{a}\left(\alpha_{1}\right), \ldots, \mathbf{a}\left(\alpha_{n}\right)\right\rangle \in \mathbf{a}(\pi)_{i} ; \mathbf{a}$ sat $\sim \phi$ in $\mathbf{A}$ at $i$ iff $\mathbf{a}$ non-sat $\phi$ in $\mathbf{A}$ at $i$; a sat $(\phi$ $\rightarrow \psi)$ in $\mathbf{A}$ at $i$ iff either $\mathbf{a}$ non-sat $\phi$ in $\mathbf{A}$ at $i$ or a sat $\psi$ in $\mathbf{A}$ at $i$; $\mathbf{a}$ sat $\forall \alpha \phi$ in $\mathbf{A}$ at $i$ iff for all $u \in D, \mathbf{a}(\alpha / u)$ sat $\phi$ in $\mathbf{A}$ at $i$; a sat $\forall \pi^{n} \phi$ in $\mathbf{A}$ at $i$ iff for all $X \in F_{n}, \mathbf{a}\left(\pi^{n} / X\right)$ sat $\phi$ in $\mathbf{A}$ at $i$; $\mathbf{a}$ sat $\forall^{k} \pi \phi$ in $\mathbf{A}$ at $i$ iff for all $u \in D$, for all $X \in K_{u}, \mathbf{a}(\pi / X)$ sat $\phi$ in $\mathbf{A}$ at $i$; and $\dot{\mathbf{a}}$ sat $\square \phi$ in $\mathbf{A}$ at $i$ iff for all $j \in I$, if $i R j$, then a sat $\phi$ in $\mathbf{A}$ at $j$.

A wff is valid in an NK-model $\mathbf{A}$ iff it is satisfied in $\mathbf{A}$ by every assignment in $\mathbf{A}$ at every possible world of $\mathbf{A}$. A $w f f$ is universally valid iff it is valid in every NK-model.

It is clear that $(K 1)$ and $(K 6)$ are valid in every $N K$-model because of clause (4) parts (iii) and (iv); (K3) is valid because of clause (4) part (ii); (K5) is valid because of clause (4) part (i); and (K2), (K4) are valid by definition of the indexed set $\left\langle K_{u}\right\rangle_{u \in D}$.

\section{Completeness:}

Besides the rule $(S)$ of substitution, the rules of inference are modus ponens, necessitation, and universal generalization of either an individual or a predicate variable. The axioms of the minimal system include all the modal axioms of $S 4$, the mixed modal principles $(K 1)-(K 5)$, axioms for standard first order logic with identity ${ }^{10}$ together

${ }^{10}$ Besides Leibniz' law and the reflexive law of self-identity, it is assumed that

$$
\forall x \forall y[\diamond x=y \rightarrow \square x=y]
$$

is also an axiom. 
with:

$$
\begin{aligned}
& \forall F^{n} \square \exists G^{n}(F \equiv G) \\
& \forall F^{n}(\phi \rightarrow \psi) \rightarrow\left(\forall F^{n} \phi \rightarrow \forall F^{n} \psi\right) \\
& \phi \rightarrow \forall F^{n} \phi \text { if ' } F^{n} \text { ' does not occur free in } \phi
\end{aligned}
$$

It is easily seen that every theorem generated from these axioms and inference rules is universally valid. It can also be shown, however, that every wff which is universally valid is a theorem of the minimal system. In addition, the system can be shown to be complete in the stronger sense that every consistent set of $w f f s$ is simultaneously satisfiable at some possible world in some NK-model. Similar completeness theorems hold for the extensions obtained by adding $(K 7)-(K 11)$ together with the above additions to clause (4) of the definition of an $N K$-model.

\section{REFERENCES}

[1] Carnap, R. "On the Use of Hilbert's $\epsilon$-Operator in Scientific Theories." In Essays on the Foundations of Mathematics, Dedicated to A. A. Fraenkel. Edited by Y. Bar-Hillel, et al. Jerusalem: Magnes Press, 1961. Pages 156-164.

[2] Cocchiarella, N. "Logical Atomism, Nominalism, and Modal Logic." Synthese 31 (1975): 23-62.

[3] Hintikka, J. Time and Necessity. London: Oxford University Press, 1973.

[4] Parsons, T. "Essentialism and Quantified Modal Logic." Philosophical Review 78 (1969): 35-52.

[5] Quine, W. "Three Grades of Modal Involvement." Proc. XIth International Congress of Philosophy, Brussells, 1953, vol. 14. Reprinted in Ways of Paradox. New York: Random House, 1966. Pages 156-174.

[6] Sellars, W. "Abstract Entities." Review of Metaphysics 16 (1963): 627-671. 